\title{
LHD REMOTE MONITORING AND MANAGEMENT BASED ON DELAYIDESRUPTION TOLERANT NETWORK
}

\author{
Shigeomi Nishigaki \\ Kick, Co., Ltd., Tokyo, Japan \\ sleepingbear@,c2mp.com \\ Katsutoshi Saibara \\ Kick, Co., Ltd., Kochi, Japan \\ saibara@,c2mp.com \\ Shigeo Kitahara \\ Kumagai Gumi Co., Ltd., Tokyo, Japan \\ skitahar@ku.kumagaigumi.co.jp \\ Hideaki Satoh \\ Kumagai Gumi Co., Ltd., Tokyo, Japan \\ hsatoh@,ku.kumagaigumi.co.jp \\ Makoto Hasegwa \\ Hitachi Construction Machinery Co., Ltd., Tokyo, Japan \\ m.hasegawa.nk@hitachi-kenki.com \\ Hiroshi Ogura \\ Hitachi Construction Machinery Co., Ltd., Ibaraki, Japan \\ h.ogura.qs@hitachi-kenki.com \\ Fujio Matsuda \\ Hitachi Construction Machinery Co., Ltd., Tokyo, Japan \\ f.matsuda.kf@hitachi-kenki.com
}

\begin{abstract}
This paper presents the Load-Haul-Dump (LHD) remote monitoring and management system based on delay/disruption tolerant network, which aims to manage a fleet to ensure reliable haulage operations at points on construction in hard environment. The fleet means the complement of construction machines, dump trucks, and workers that are working together. Connectivity on the Internet primarily relies on wired links, including the wired telephone network and cellular phone network. When constructing a field network, however, we often face a particular communication region outside of the Internet and a transmission of data delayed. This paper gives arguments about some of the ways to handle data communication that suffers delay or disruption in such a hard environment. In addition, are reported two case studies on the deployment of the LHD remote monitoring and management system to accommodate mobility of data, in order to manage construction machines and dump trucks in a haul work.
\end{abstract}

KEYWORDS: LHD, remote monitoring on real time basis, event detection, and delay/disruption-tolerant. 


\section{MOTIVE}

This paper presents the Load-Haul-Dump (LHD) remote monitoring and management based on delay/disruption-tolerant network, which aims to manage a fleet to ensure reliable haulage operations at points on construction in hard environment. The fleet means the complement of construction machines, dump trucks, and workers that are working together. Examples of materials hauled here include borrow soil, shot rock, aggregate, concrete, and other materials.

The LHD remote monitoring and management system consists of the collaborative construction management professional (hereafter refer to as c2mProfessional) and the Points on Construction system (hereafter refer to as POC). The c2mProfessional system is a comprehensive, web-enabled management system to support day-to-day construction management. It has a variety of functions to manage information on day-to-day construction activities. Relevant functions of those might be chosen and assembled as being proactive to customer's needs and job-site conditions, and would be provided as ASP service for construction authorities, contractors, subcontractors, and others. The POC is a site network tracking system for mobile entities such as construction machines, dump truck, workers, materials, and so on.

So far, the POC has been and is being studied so as to remotely monitor operations on job sites and to provide resident engineers and supervisors with information on construction efficiency for loading, hauling and dumping of borrow soil (Nishigaki et al., 2008). Figure 1 shows an overview of the POC deployment.

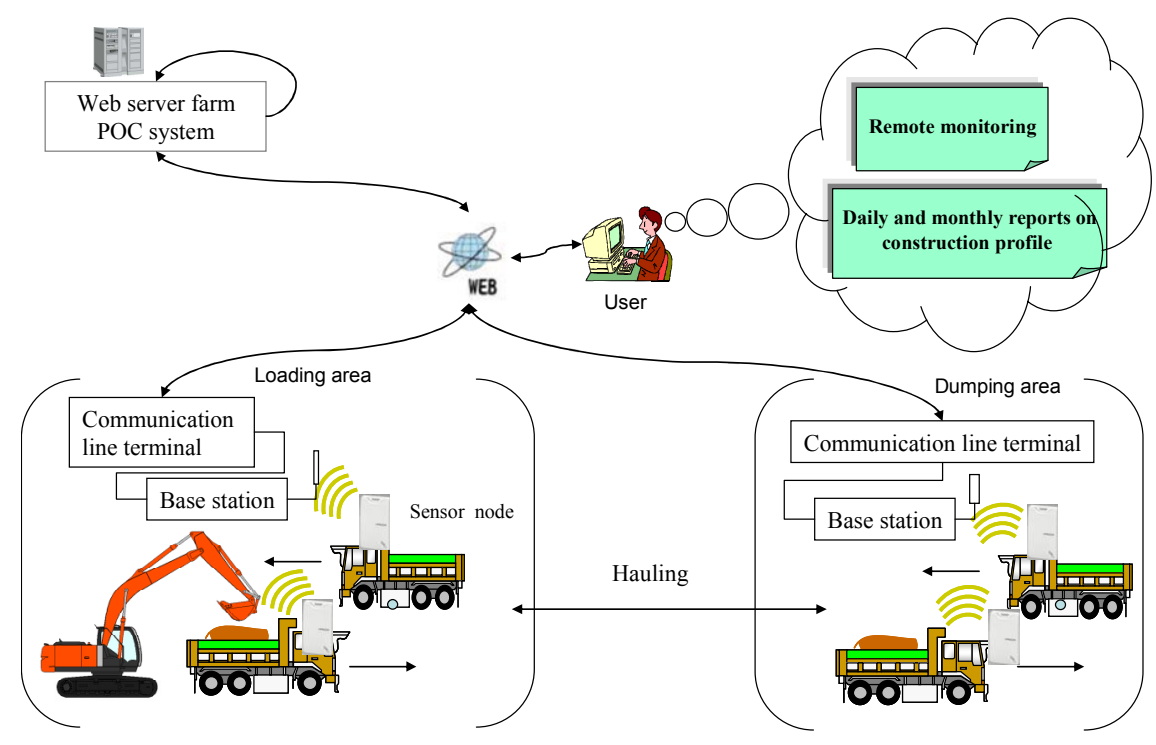

Figure 1: Overview of the POC Deployment

In the above deployment, connectivity on the TCP/IP based Internet primarily relies on endto-end communication through wired links, including wired telephone network and cellular phone network. When constructing a field network, however, we often face a particular communication region outside of the Internet and a transmission of data delayed. This paper presents arguments about some of the ways to handle data communication that suffers delay or disruption in such a hard environment, and developed the LHD remote monitoring and 
management system to accommodate mobility of data in order to manage backhoes operated and dump trucks travelled to haul materials.

\section{SCHEME}

\section{Configuration}

Figure 2 gives an overview of the configuration of the LHD remote monitoring and management system. As mentioned before, the LHD remote monitoring and management system consists of the $\mathrm{c} 2 \mathrm{mProfessional}$ and the POC.

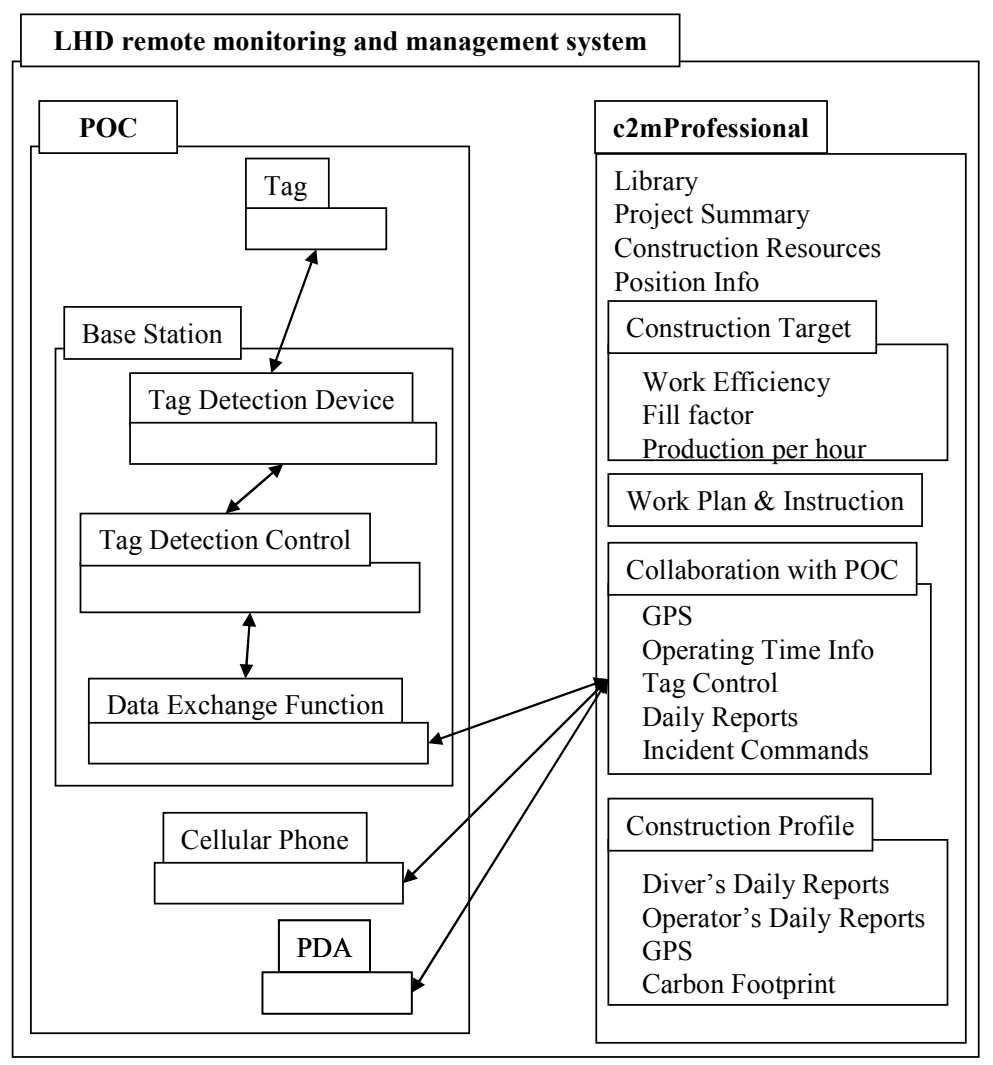

Figure 2: Overview of the LHD Remote Monitoring and Management System

This system is deployed in the three layers as shown in Figure 3. Having said that, the POC means a site network tracking system for mobile entities, and could plays a large role in construction field. In the POC layer, are used a number of transmitting and receiving base station and nodes (that is, active tag in this study), web camera, GPS Logger, cellular phone, PDA, satellite antenna, which connect through mediums of wireless communication, wired link communication, or satellite communication with the c2mProfessional. In the layer of operation and maintenance, are operated and maintained the c2mProfessional and the Globale-Service. The Global-e-Service is a multi-language internet-based back office management system in maintenance and service applications for construction machinery. It is able to monitor and control machine production with a satellite communication system, GPS 
positioning system, and on-board computer, and is served worldwide (Nishigaki et al., 2008). In the data utilization layer, the data captured by the POC are aggregated, converted, or reformatted into a uniform structure, which is subsequently displayed on real time basis, and edited as the construction profile within a reasonable timeframe.

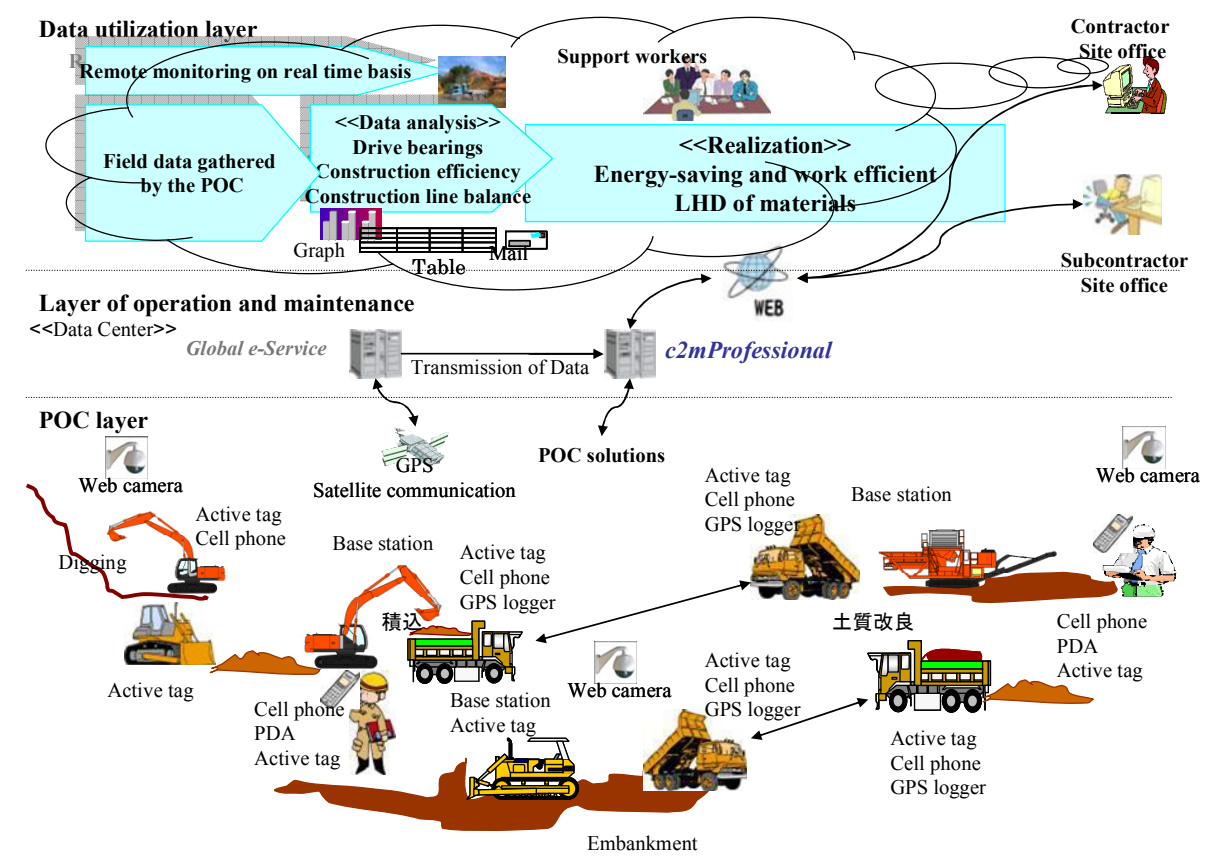

Figure 3: Overview of the LHD Remote Monitoring and Management System deployed to Earth Working and Haulage of Borrow Soil

\section{Event Detection}

In this study, an event is defined as a significant occurrence or activity related to loading hauling, dumping, spreading, and compacting, which are operations in earthwork. There are two event detection approaches to identify the events through a process of earthwork. The first is a sensor-based event detection that utilizes wireless sensor medium to detect a significant occurrence or activity automatically. The other is an operator intervention approach to recognize it manually. If possible, the beginning and end of occurrence could be automatically detected by wireless sensor medium, and otherwise recognized by a button pressed event, that is, pushing predetermined function key by operator. Figure 4 shows the concept of the event detection approaches.

In the sensor-based event detection approach, are used wireless LAN-based devices that consist of active tags and base stations with about $100 \mathrm{~m}$ effective range. The each dump truck carries an active tag. The base stations are put in position on the side of the entrance to the loading and the dumping spots, respectively. The base station successively receives signals from an active tag with the dump truck that comes into the effective cover range. The signal includes the ID of the active tag and, if necessary, the code of material hauled Then the basic station automatically sends the c2mProfessional via the Internet its own ID, the active tag ID received and the time stamps of the ingress to and egress from the spot, that is, its effective radio range. 


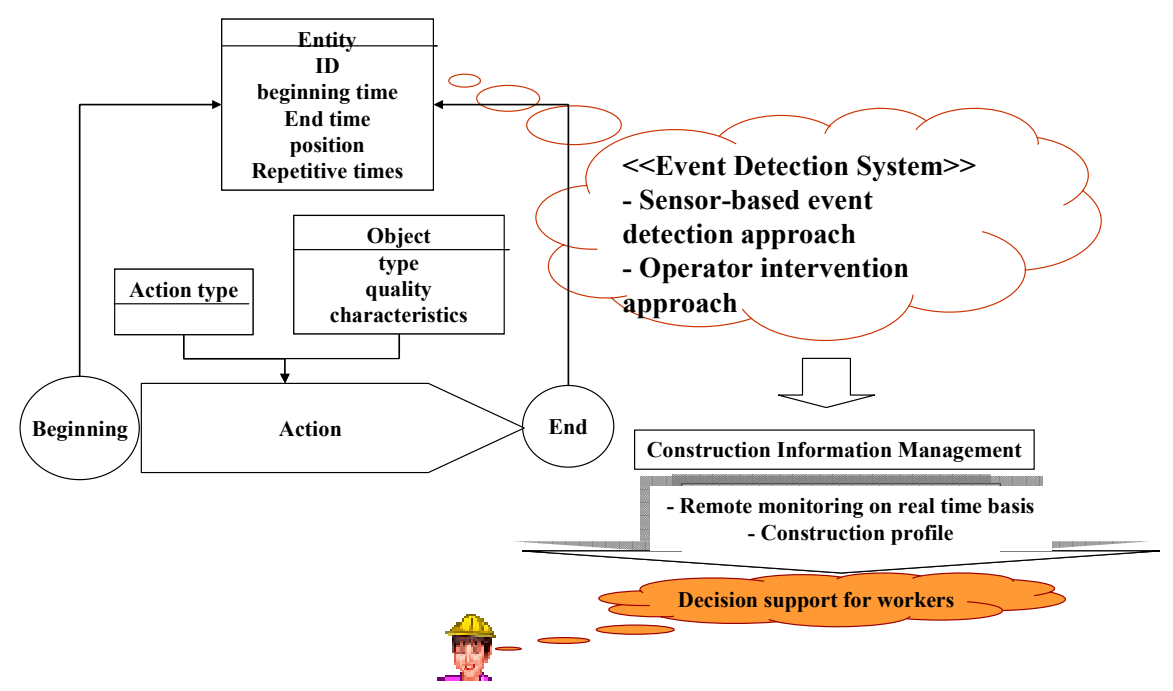

Figure 4: Event Detection Approaches

Facing the construction area difficult of approach according to something of avalanche of earth and rocks, land slide, or rock falls, unmanned construction, that is, tele-operation of construction machines, is often introduced to a disaster recovery or reconstruction. In the unmanned construction, tele-operators would ensure concurrent and subsequent reliable operation of their construction machines as watching monitors that display the visual work scenes in the time and space domain. The visual work scenes are fed back by cameras mounted on the construction equipment and the construction machine. Since it is very danger for human to manually put the wireless sensor medium in position on the above construction area, the operator intervention approach is often introduced to recognize events related to tele-operated construction machines. Here, tele-operators would push the predetermined function key in their keyboard if and when they should confirm the beginnings or the ends of occurrences on the monitor.

The POC temporarily stores the captured data into its data base and automatically sends them to the $\mathrm{c} 2 \mathrm{mProfessinal}$. Subsequently, the $\mathrm{c} 2 \mathrm{mProfessional} \mathrm{stores} \mathrm{the} \mathrm{received} \mathrm{data} \mathrm{into} \mathrm{its}$ data base, and utilize them to produce daily and monthly reports on construction profile for future validation. The construction profile here is defined as a set of data to vision characteristics of phenomena being generated along with construction in progress and as indices to show their patterns (Nishigaki et al., 2005).

\section{Event Detection Algorithm}

Having said that, in the sensor-based event detection approach, are used wireless LAN-based devices that consist of active tags and base stations. The base station has the radio range that is something of the order of $100 \mathrm{~m}$. The base station could successively receive signals from the active tag while it stays in the effective radio range of the base station. The first detection time of the signals is regarded as the beginning time when the mobile entity came into the effective radio range. The last time of the signals identified is regarded as the end time when the mobile entity went away from the effective radio range. Problem here is how to recognize the last one of the signals received. Figure 5 shows an event detection sequence in the sensor-based event detection approach. 


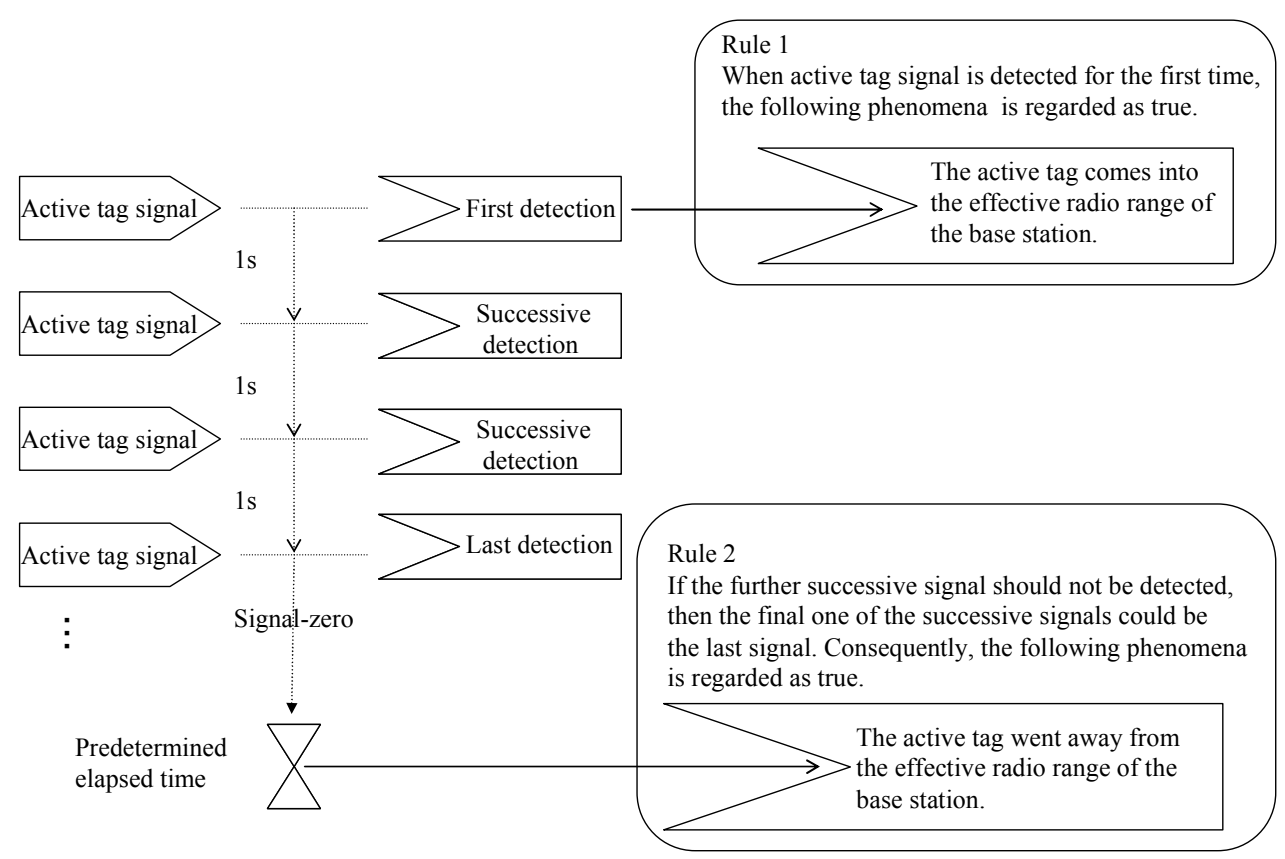

Figure 5: Event Detection Sequence in the Sensor-based Event Detection approach

If and when the duration being void of successive signals exceeds the predetermined elapsed time, then the last signal received is regarded as the time when the active tag went away from the effective radio range of the base station. Another said way, the dump truck came in the work spot at the time when the signal was captured at the first time by the base station, and on the other hand it went out of the work spot at the time when the last signal received.

As previously described, the operator intervention approach is introduced to tele-operation of construction machines in such a hard environment. In the operator intervention approach, in order to recognize events related to dumping by off-highway dump truck, spreading by bulldozer, or compacting by vibration roller, tele-operators would push the predetermined function keys in their keyboards, if and when they should confirm the beginnings or the ends of the occurrences displayed on the monitor. Pushing the function key results in the button pressed event, which shall be automatically sent with the timestamp to the c2mProfessional via satellite communication, mobile communication or the like.

\section{Store and Forward Method}

Facing a particular communication region outside of the Internet, the store and forward method would be used in order to gather field data of events, materials hauled, positions, and so on. The store and forward method makes it possible to support interconnecting communication network with delay/disruption paths between a base station isolated outside of the Internet and another base station that plays a role in a gateway to the Internet by intermittently contacting active tags in motion. An overview of the store and forward method is shown in Figure 6.

Suppose that there are two base stations. The first is emplaced at entrance of loading site where is outside of the Internet. The other is put in position on entrance of dumping site where it is possible to connect with the Internet. Dump trucks with active tags carry 
materials from the loading site to the dumping site. At the egress from the loading site, the active tags receive data of the base station ID, code of material hauled, egress time, and the last ingress time. The dump trucks drive along the designated route in order to haul materials to the destination, that is, the dumping site. At the ingress into the dumping site, the active tags emit their carried data and their own ID to the base station there. And then this base station automatically sends the received data with its own ID to the c2mProfessional via the Internet. After dumping, the dump trucks return to the loading site. At the ingress into the loading site, the active tags emit signal of their own ID to the base station there.

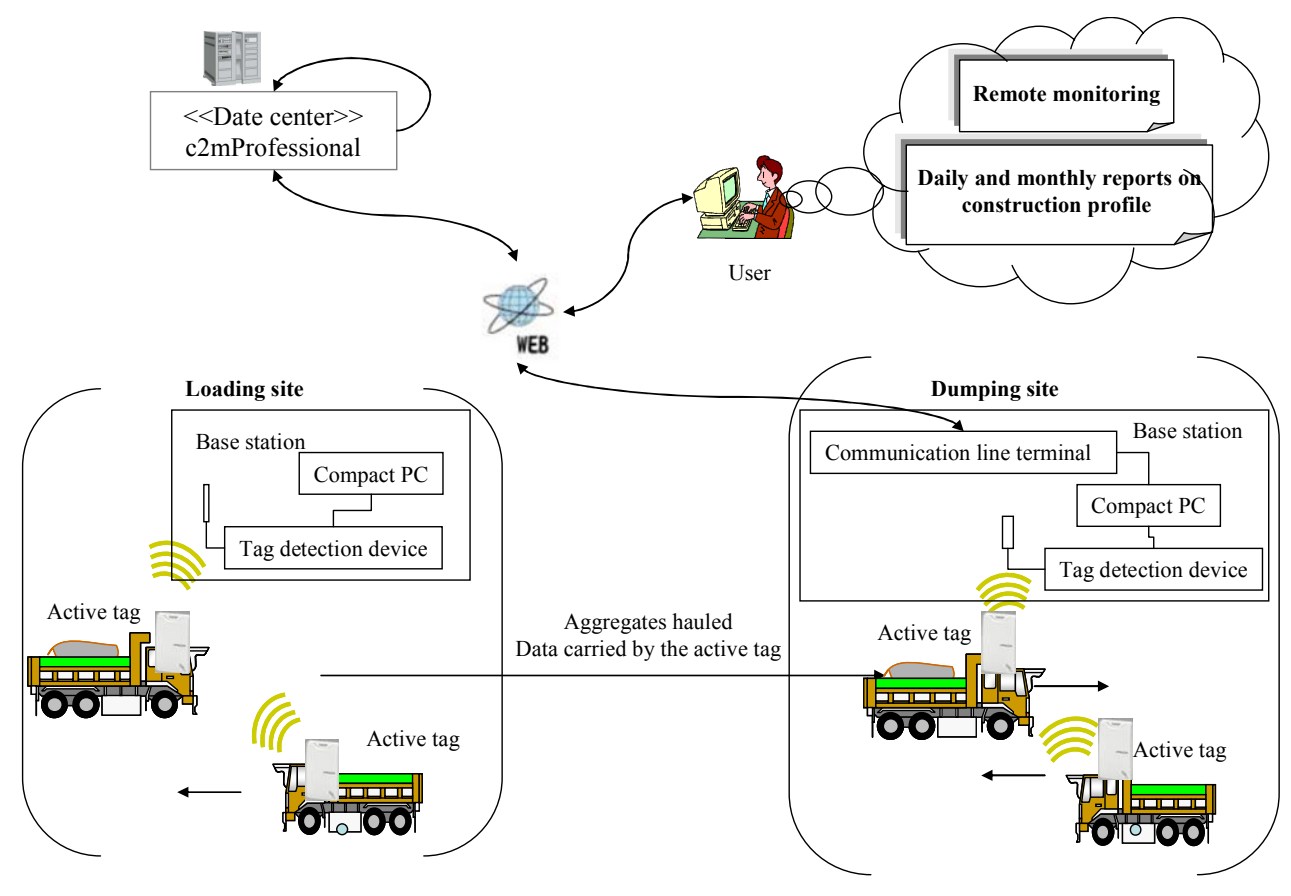

Figure 6: Overview of the Store and Forward Method

\section{CASE STUDIES}

\section{Aggregates Haulage}

Figure 7 gives an overview of the field experiment of the store and forward method, which is applied to aggregates hauled from aggregation production plant to concrete batching plant at dam site.

Aggregates here are composed of three globular domains, G1 $(80-40 \mathrm{~mm}), \mathrm{G} 2(40-20 \mathrm{~mm})$ and G3 $(20-5 \mathrm{~mm})$. The base station at the aggregation production plant is isolated from the Internet. On the contrary, the base station at the concrete batching plant could be connected with the Internet. The active tags, which dump trucks carry, are independently most of time in motion between the two base stations and in the meanwhile remain disconnected from the two. The active tags play a role of intermediate storage place for the data to be communicated between the two. The active tags might make contact and send the base station the data forward, and receive the base station ID backward, when they might be close 
enough to communicate with each other. Subsequently, the base station at the dumping site automatically sends the received data with its own ID forward to the c2mProfessional.

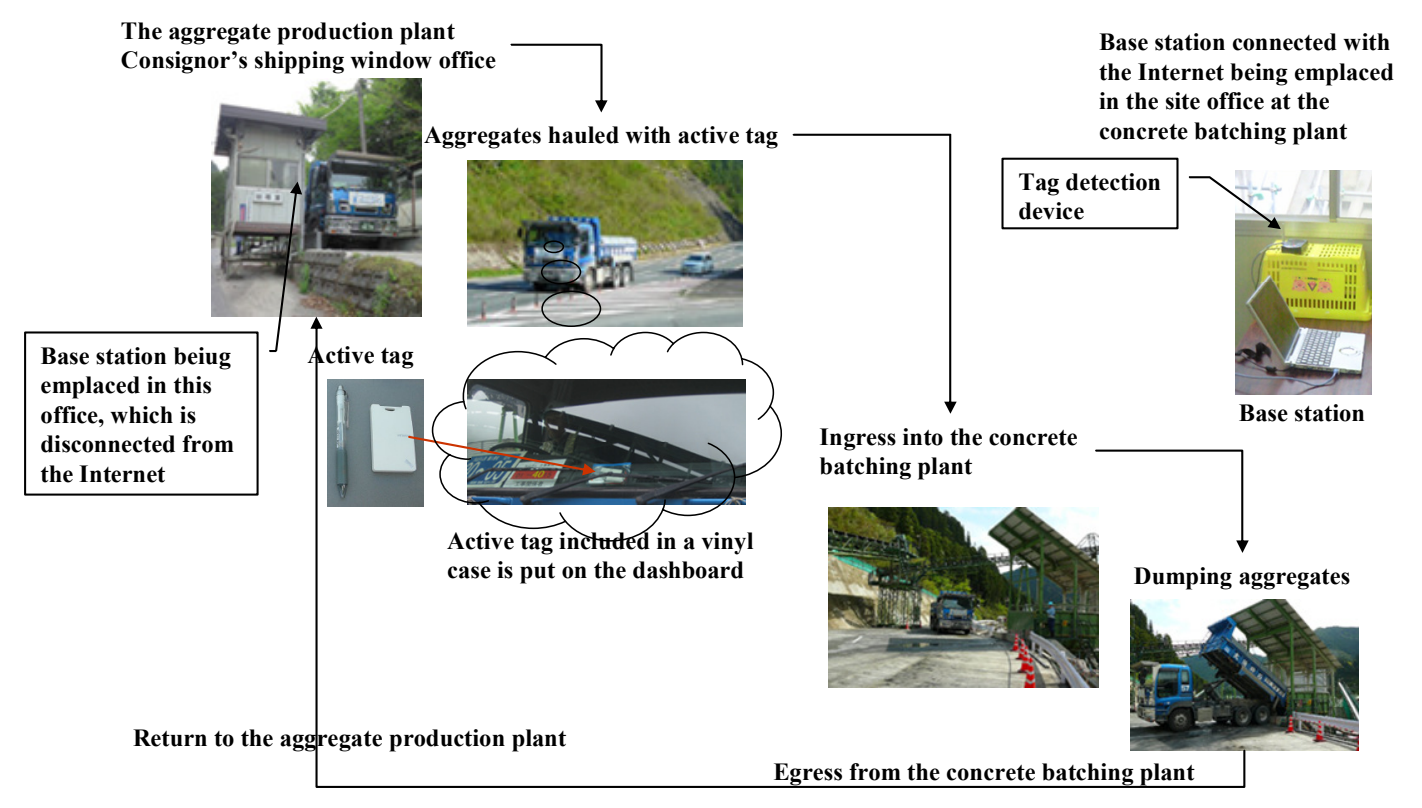

Figure 7: Overview of the Field Experiment of the Store and Forward Method

\section{No-slump Concrete Haulage}

The LHD remote monitoring and management system is deployed to haul no-slump concrete for a check dam construction against avalanche of earth and rocks. Figure 8 gives an overview of this deployment. The no-slump concrete is hauled by 10 ton dump trucks from ready-mixed concrete batching plant to the spot where it is transferred to off-highway dump trucks as shown in Figure 9. Subsequently, the off-highway dump trucks haul the no-slump concrete to the designated dumping spot. In addition, a bulldozer spreads the no-slump concrete, and subsequently a vibration roller compacts. Here, two off-highway dump trucks, a bulldozer and a vibration roller are tele-operated as shown in Figure 9.

The base station at the ready-mixed concrete batching plant is connected via mobile communication with the Internet. On the other hand, the base station at the transfer spot is connected via a satellite communication with the Internet. The beginning and end of loading and dumping to transfer no-slump concrete are captured by the sensor-based event detection system. On the other hand, in this tele-operation, the operator intervention approach is introduced to recognize occurrences related to dumping by the off-highway dump truck, spreading by the bulldozer, or compacting by the vibration roller.

The event detection system here is capable of identifying events within a mount of short time through a process of the no-slump concrete transportation and placement. The POC including this event detection system automatically sends the c2mProfessional the data related to the events identified. Furthermore, the c2mProfessional will edit and summarise the data into a construction profile within a reasonable timeframe. 


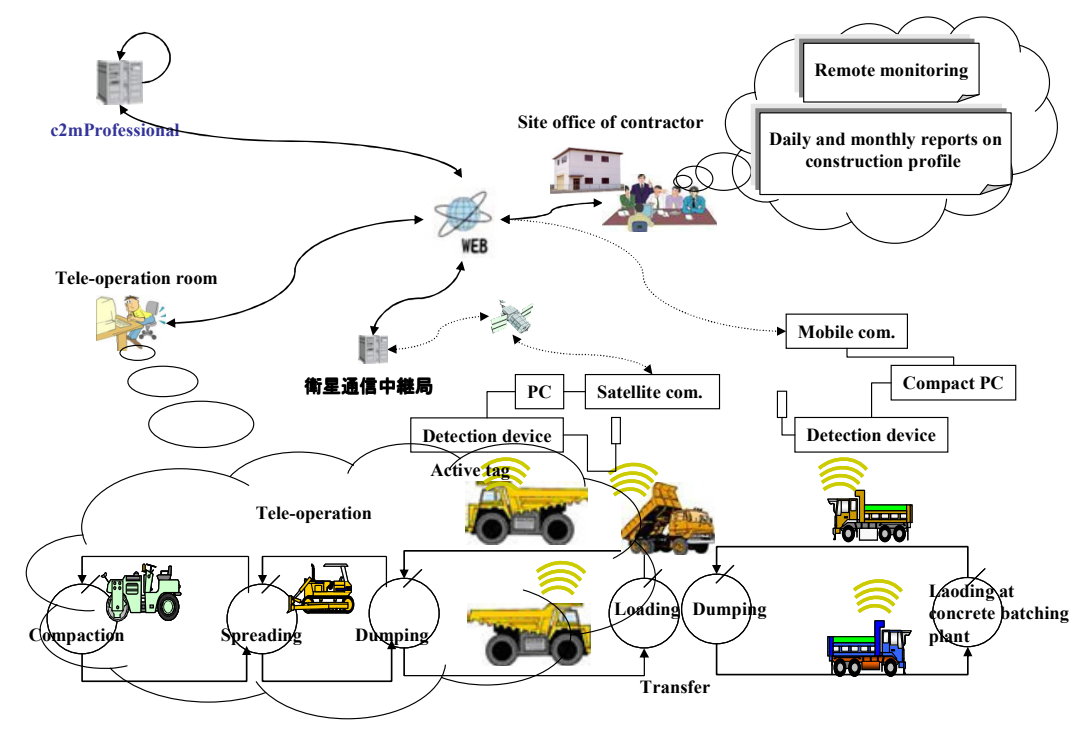

Figure 8: Overview of the Deployment of the LHD Remote Monitoring and Management System to No-slump Concrete Haulage

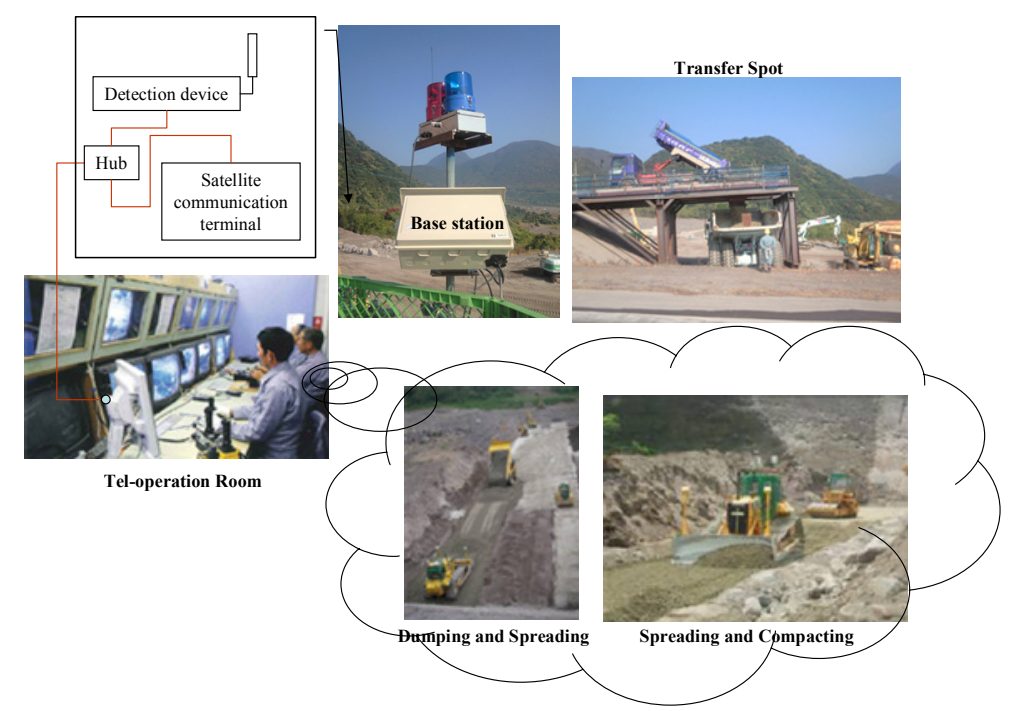

Figure 9: Transfer Spot of No-slump Concrete from 10 ton Dump Truck to Off-highway Dump Truck and Tele-operation of Construction Machines

Figure 10 shows an example of the remote monitoring display on the PC provided by the c2mProfessional. The information on the display gives the current construction profile at one-minute intervals. Also, the information on the remote monitoring can be confirmed by a cellular phone. As watching the remote monitoring display, tele-operators, supervisors and resident engineers are able to recognize early coloured warnings based on the imminent left time allowable to place the no-slump concrete. The early coloured warnings consist of the coloured classes such as "Blue" means the completion, "No-colored" the left time equal to or more than one hour, "Yellow" the left time less than one hour, and "Red" the left time less than thirty minutes. 


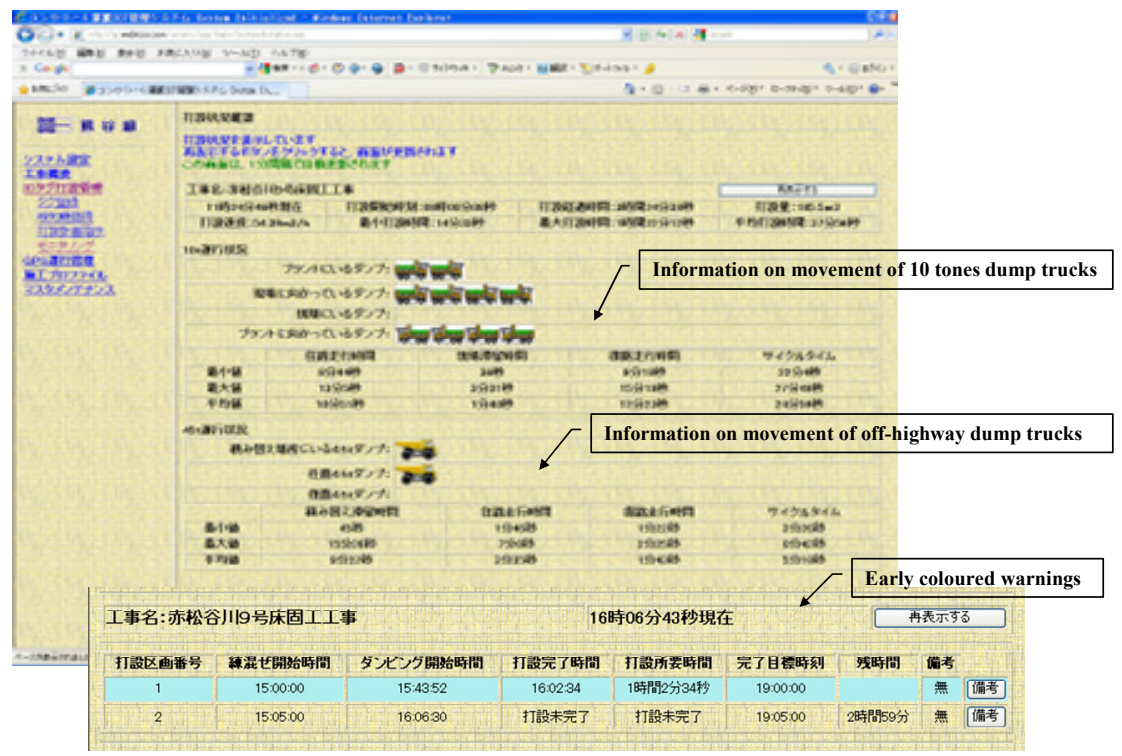

Figure 10: Example of Remote Monitoring Display

\section{REMARKS}

The LHD remote monitoring and management system gives a detailed visibility into appearances and motions in loading, haulage and dumping, and enables the decision-makers to take quick actions with the objective of increasing construction efficiency and energysaving on daily duty-cycle.

Further works are shown as follows:

- Alive monitoring system to ensure whether wireless senor medium remains functional or not, and

- Prevention method against human errors related to loading material and dumping position, for examples, mistaken material and wrong position.

\section{REFERENCES}

S. Nishigaki, K. Saibara, F. Matsuda, H. Ogura (2008) Points On Construction,, 25th ISARC 2008, Vilnius, Lithuania, 796-803.

S. Nishigaki, H. Ogura, et al. (2005) Study on Framework of Construction Profile for Collaboration and Intelligent Construction, Journal of Applied Computing in Civil Engineering, 14, 287-298 